\title{
Prediction of Occult Lymph Node Metastasis by Metabolic Parameters in Patients with Clinically NO Esophageal Squamous Cell Carcinoma
}

\author{
Seung Hwan Moon ${ }^{1}$, Ho Seong Kim¹ ${ }^{1}$, Seung Hyup Hyun ${ }^{1}$, Yong Soo Choi ${ }^{2}$, Jae Ill Zo², Young Mog Shim², \\ Kyung-Han Lee ${ }^{1}$, Byung-Tae Kim ${ }^{1}$, and Joon Young Choi ${ }^{1}$ \\ ${ }^{1}$ Department of Nuclear Medicine, Samsung Medical Center, Sungkyunkwan University School of Medicine, Seoul, Republic of \\ Korea; and ${ }^{2}$ Department of Thoracic Surgery, Samsung Medical Center, Sungkyunkwan University School of Medicine, Seoul, \\ Republic of Korea
}

\begin{abstract}
The aim of this study was to investigate the value of ${ }^{18} \mathrm{~F}-\mathrm{FDG}$ parameters of the primary tumor in predicting occult lymph node metastasis in patients with clinically NO esophageal squamous cell carcinoma. Methods: The study comprised 143 consecutive patients (mean age $\pm \mathrm{SD}, 63.9 \pm 8.6 \mathrm{y}$; range, 31.8-81.2 y) from May 2003 to January 2010 who had clinically NO esophageal squamous cell carcinoma based on preoperative imaging studies including chest CT, ${ }^{18} \mathrm{~F}-\mathrm{FDG}$ PET/CT, and endoscopic ultrasound. We measured maximum standardized uptake value $\left(S_{U} V_{\text {max }}\right)$, mean SUV $\left(S_{\text {mean }}\right)$, total lesion glycolysis (TLG), and metabolic tumor volume (MTV) of the primary tumor and analyzed the relationship between clinicopathologic variables including PET parameters and occult lymph node metastasis using a logistic regression model. Results: Univariate analysis indicated that clinical T classification, SUV $\max$, SUV $_{\text {mean }}$, MTV, TLG, and longitudinal diameter of tumor were significant risk factors associated with occult lymph node metastasis. Optimal thresholds were cT2-4, SUV max $\geq 4.8$, SUV mean $\geq 3.2$, MTV $\geq 5.5 \mathrm{~cm}^{3}, T L G \geq 220$, and diameter $\geq 3.8 \mathrm{~cm}$. After multivariate analysis, the logistic regression model revealed that clinical T classification (hazard ratio [HR], 4.6; 95\% confidence interval [Cl], 1.7-12.4; $P=0.003)$ and SUV $_{\max }(\mathrm{HR}, 3.5 ; 95 \% \mathrm{Cl}, 1.3-9.2 ; P=0.012)$ were independent risk factors. The combination of SUV $\mathrm{max}_{\max }$ and clinical T classification (HR, 13.2; 95\% Cl, 5.4-31.9; $P<0.001)$ was a significantly better powerful risk factor for occult lymph node metastasis than SUV $\mathrm{max}_{\text {max }}$ or clinical T classification alone. Sensitivity, specificity, positive predictive value, and negative predictive value of the combination of clinical T classification and SUV $\max$ were $73.0 \%, 81.5 \%$, $60.0 \%$, and $89.7 \%$, respectively. Conclusion: SUV $V_{\max }$, combined with clinical $\mathrm{T}$ classification, may be useful for predicting occult lymph node metastasis in patients with clinically NO squamous cell carcinoma of the esophagus.
\end{abstract}

Key Words: esophageal cancer; ${ }^{18} \mathrm{~F}-\mathrm{FDG}$; PET/CT; lymph node; occult metastasis; SUV $V_{\max }$

J Nucl Med 2014; 55:743-748

DOI: 10.2967/jnumed.113.130716

Received Aug. 11, 2013; revision accepted Nov. 20, 2013.

For correspondence or reprints contact: Joon Young Choi, Department of Nuclear Medicine, Samsung Medical Center, Sungkyunkwan University School of Medicine, 50 Irwon-dong, Gangnam-gu, Seoul, 135-710 Republic of Korea.

E-mail: jynm.choi@samsung.com

Published online Apr. 3, 2014.

COPYRIGHT (c) 2014 by the Society of Nuclear Medicine and Molecular Imaging, Inc.
$\mathbf{T}$ he accurate detection of regional lymph node metastasis in esophageal cancer is important for the selection of the proper treatment strategy. The presence of regional lymph node metastasis is particularly critical when making decisions about treatment, especially when tumor invasion is limited to submucosa of the esophagus. Current evaluation methods for preoperative tumor classification include multiimaging modalities such as CT, endoscopic ultrasonography (EUS), and ${ }^{18}$ F-FDG PET/CT (1). However, current imaging modalities have limited capabilities, and diagnostic performance for the detection of regional lymph node metastases is incomplete (2). Despite comprehensive assessment using multiimaging modalities, a considerable percentage of patients with clinically NO esophageal cancer are found to have regional lymph node metastasis after esophagectomy. The prevalence rate of occult lymph node metastasis ranges from $11 \%$ to $56 \%$ (3).

${ }^{18} \mathrm{~F}$-FDG PET/CT has an advantage over conventional imaging modalities in that it provides quantitative information on metabolic activity of the tumor (4). Quantified metabolic activity measured by ${ }^{18}$ F-FDG PET can reflect tumor burden and disease activity; therefore, it is useful for both predicting prognosis and assessing treatment response in the field of clinical oncology (5-7). Recently, it was reported that metabolic PET parameters were independent risk factors associated with occult lymph node metastasis in patients with non-small cell lung cancer who were diagnosed as N0-1 by preoperative staging work-up and in patients with clinically nodenegative squamous cell carcinoma of the tongue $(8,9)$. This finding suggests a potential benefit of ${ }^{18} \mathrm{~F}$-FDG $\mathrm{PET} / \mathrm{CT}$ in preoperative assessments of lymph node metastasis in cancer patients who have been diagnosed as clinically node-negative.

The purpose of this study was to investigate whether ${ }^{18} \mathrm{~F}$-FDG PET parameters of the primary tumor have the potential to predict occult lymph node metastasis in patients with clinically NO esophageal cancer.

\section{MATERIALS AND METHODS}

\section{Patients}

We retrieved data on all consecutive patients with esophageal cancer who underwent ${ }^{18}$ F-FDG PET/CT for initial staging from May 2003 to January 2010 by searching the institutional medical database. Patients were included if they had a pathologically proven esophageal squamous cell carcinoma with clinically NO classification, based on preoperative studies including chest CT, ${ }^{18} \mathrm{~F}-\mathrm{FDG}$ PET/CT, and EUS. To be included, patients also had to have undergone esophagectomy with lymph 
node dissection without neoadjuvant treatment. Patients with concurrent cancer at the time of diagnosis or uncontrolled diabetes or high blood glucose level $(>150 \mathrm{mg} / \mathrm{dL})$ during the PET/CT scan were excluded.

Study patients were selected from a total of 892 patients with squamous cell carcinoma who underwent PET/CT for initial staging work-up. After exclusion of 365 (40.9\%) patients who did not undergo EUS or who did not cooperate during the EUS, 270 (30.3\%) patients with $\mathrm{cN} 1-3$ disease, $78(8.7 \%)$ patients who had endoscopic mucosal resection or preoperative therapy, $12(1.3 \%)$ patients who had concurrent cancer, $23(2.6 \%)$ patients who had uncontrolled diabetes or high blood glucose level, and $1(0.1 \%)$ patient with PET/CT data loss, 143 (16.0\%) patients were finally included for analysis.

The institutional review board our institution approved this retrospective study, and the requirement to obtain informed consent was waived.

\section{PET/CT Imaging}

Patients were instructed to fast for at least $6 \mathrm{~h}$ before the PET/CT scan. Blood glucose levels were measured before injection of ${ }^{18} \mathrm{~F}$-FDG in all patients. PET/CT imaging was performed using 1 of 2 dedicated PET/CT scanners (Discovery LS for 89 subjects or Discovery STe for 96 subjects; GE Healthcare) without intravenous or oral contrast material.

When the Discovery LS scanner was used, whole-body CT was performed with a continuous spiral technique with an 8-slice helical CT (140 keV; 40-120 mA; section width, $5 \mathrm{~mm}$ ) at $60 \mathrm{~min}$ after injection of ${ }^{18} \mathrm{~F}-\mathrm{FDG}(5.5 \mathrm{MBq} / \mathrm{kg})$. After the CT scan, an emission scan was obtained from head to toe for 4 min per frame in 2-dimensional mode. Attenuation-corrected PET images $(4.3 \times 4.3 \times 3.9 \mathrm{~mm})$ were reconstructed from the CT data using an ordered-subset expectation maximization algorithm (28 subsets, 2 iterations). When the Discovery STe scanner was used, whole-body CT was performed with a continuous spiral technique with 16-slice helical CT (140 keV; 30$170 \mathrm{~mA}$; section width, $3.75 \mathrm{~mm}$ ) at $60 \mathrm{~min}$ after injection of ${ }^{18} \mathrm{~F}$ FDG. After the CT scan, an emission scan was obtained from the thigh to head for $2.5 \mathrm{~min}$ per frame in 3-dimensional mode. Attenuationcorrected PET images $(3.9 \times 3.9 \times 3.3 \mathrm{~mm})$ were reconstructed from the CT data using a 3-dimensional ordered-subset expectation maximization algorithm (20 subsets, 2 iterations).

\section{Measurements of PET Parameters}

Two experienced nuclear medicine physicians reviewed all PET/CT images. Metabolic and volumetric parameters were measured using Volume Viewer software (GE Healthcare). Metabolic tumor volume (MTV) was defined as the total tumor volume segmented by the threshold standardized uptake value (SUV). Mediastinal blood-pool activity was used as a threshold for determining the volume-of-interest (VOI) boundary. To determine the threshold using the mediastinal blood pool, a VOI consisting of $5 \times 5 \times 1$ voxels was manually drawn at the aortic arch. The mean SUV ( $\left.\mathrm{SUV}_{\text {mean }}\right)$ plus 2 SDs of the VOI in the aortic arch was chosen as the threshold SUV for the selected measurable tumors. VOIs of the tumors were automatically generated using the threshold SUV. The software calculated the maximum SUV (SUVmax), $\mathrm{SUV}_{\text {mean }}$, and MTV of the entire tumor. Total lesion glycolysis (TLG) was obtained by multiplying the $\mathrm{SUV}_{\text {mean }}$ by the number of voxels $(10)$.

\section{Lymph Node Grouping}

All visible and palpable lymph nodes in the surgical field were dissected thoroughly by experienced thoracic surgeons as presented in the previous study conducted at Samsung Medical Center (11). We classified the lymph nodes into 5 categories according to lymph node region for the purpose of analysis, according to previous studies $(11,12)$. In the present study, cervical nodes included cervical and supraclavicular lymph nodes. Upper mediastinum nodes included upper paraesophageal lymph nodes, recurrent nerve lymph nodes, aortopulmonary nodes, and paratracheal lymph nodes. Middle mediastinum
TABLE 1

Clinicopathologic Characteristics of Patients with Clinically NO Esophageal Squamous Cell Carcinoma $(n=143)$

\begin{tabular}{|c|c|}
\hline Characteristic & No. of patients \\
\hline \multicolumn{2}{|l|}{ Age (y) } \\
\hline Range & $31-81$ \\
\hline Median & 64 \\
\hline \multicolumn{2}{|l|}{ Sex } \\
\hline Male & $131(91.6)$ \\
\hline Female & $12(8.4)$ \\
\hline \multicolumn{2}{|l|}{ Tumor location } \\
\hline Upper & $10(7.0)$ \\
\hline Middle & $82(57.3)$ \\
\hline Lower & 48 (33.6) \\
\hline Esophagogastric junction & $3(2.1)$ \\
\hline \multicolumn{2}{|l|}{ Histologic grade } \\
\hline Well & $16(11.2)$ \\
\hline Moderate & $111(77.6)$ \\
\hline Poor & $16(11.2)$ \\
\hline \multicolumn{2}{|l|}{ Operation } \\
\hline Two-field & $140(97.9)$ \\
\hline Three-field & $3(2.1)$ \\
\hline \multicolumn{2}{|l|}{ cT classification } \\
\hline Tis-T1 & $65(45.4)$ \\
\hline T2 & $48(33.6)$ \\
\hline T3 & $28(19.6)$ \\
\hline T4 & $2(1.4)$ \\
\hline \multicolumn{2}{|l|}{ pT classification } \\
\hline Tis-T1a & $35(24.5)$ \\
\hline T1b & $63(44.0)$ \\
\hline T2 & $18(12.6)$ \\
\hline T3-4 & 27 (18.9) \\
\hline \multicolumn{2}{|l|}{ pStage } \\
\hline $0-1$ & $86(60.1)$ \\
\hline IIA & $9(6.3)$ \\
\hline IIB & $27(18.9)$ \\
\hline III & $21(14.7)$ \\
\hline Occult lymph node metastasis & $37(25.9)$ \\
\hline $\begin{array}{l}\text { No. of dissected lymph nodes } \\
\text { (mean } \pm \text { SD) }\end{array}$ & $35.5 \pm 14.8$ \\
\hline $\begin{array}{l}\text { cLongitudinal diameter of tumor } \\
(\mathrm{cm} ; \text { mean } \pm \mathrm{SD})\end{array}$ & $2.9 \pm 1.7$ \\
\hline
\end{tabular}

$\mathrm{c}=$ clinical; $\mathrm{p}=$ pathologic.

Data in parentheses are percentages.

nodes included subcarinal nodes, middle paraesophageal lymph nodes, and hilar lymph nodes. Lower mediastinum nodes included lower paraesophageal and diaphragmatic lymph nodes. Abdominal nodes included cardiac, left gastric, and celiac lymph nodes.

\section{Statistical Analysis}

Statistical analyses were performed using commercial software (PASW Statistics 18; IBM Inc.). PET parameters and clinicopathologic characteristics were analyzed to identify risk factors for occult lymph node metastasis. Information including tumor location, histology, and histologic grade was obtained from surgical records and pathology reports. Clinical tumor depth (cT classification) and clinical longitudinal diameter of tumor were determined by EUS.

Receiver-operating-characteristic curve analysis was performed to determine the optimal cutoff values for the continuous variables that divided patients into 2 subgroups according to presence of occult lymph node metastasis. Univariate and multivariate analyses using backward 
TABLE 2

Comparison of PET Parameters Between Patients With and Without Occult Lymph Node Metastasis

\begin{tabular}{|c|c|c|c|}
\hline Parameter & Metastasis $(+)$ & Metastasis (-) & $P$ \\
\hline$S_{\text {SUax }}$ & & & $<0.001$ \\
\hline Median & 10.3 & 3.5 & \\
\hline Mean \pm SD & $9.9 \pm 6.2$ & $5.5 \pm 6.5$ & \\
\hline Range & $1.0-22.3$ & $1.0-39.8$ & \\
\hline$S U V_{\text {mean }}$ & & & 0.02 \\
\hline Median & 3.4 & 2.4 & \\
\hline Mean \pm SD & $3.3 \pm 1.3$ & $2.5 \pm 2.0$ & \\
\hline Range & $1.0-6.70$ & $1.0-15.0$ & \\
\hline $\operatorname{MTV}\left(\mathrm{cm}^{3}\right)$ & & & 0.03 \\
\hline Median & 10.2 & 2.9 & \\
\hline Mean \pm SD & $17.9 \pm 23.4$ & $9.9 \pm 20.3$ & \\
\hline Range & $0.0-104.0$ & $0.0-120.0$ & \\
\hline TLG & & & 0.14 \\
\hline Median & 640.2 & 125.1 & \\
\hline Mean \pm SD & $1,427.4 \pm 2,664.8$ & $843.6 \pm 2,224.6$ & \\
\hline Range & $0.0-12,243.3$ & $0.0-13,107.0$ & \\
\hline
\end{tabular}

stepwise logistic regression modeling were performed to identify the risk factors associated with occult lymph node metastasis. In addition, subgroup analysis was performed in the cT1N0 and cT2N0 patients group separately. The McNemar test was used to compare the accuracy of factors significance on multivariate analysis for predicting occult lymph node metastasis. Continuous variables were expressed as mean \pm SD. All tests were 2 -sided, and $P$ values less than 0.05 were considered statistically significant.

\section{RESULTS}

\section{Patient Characteristics}

The patients' clinicopathologic characteristics are summarized in Table 1. The mean time interval between ${ }^{18} \mathrm{~F}-\mathrm{FDG}$ PET/CT and operation was $11 \pm 7 \mathrm{~d}$, ranging from 1 to $30 \mathrm{~d}$. The incidence of occult lymph node metastasis was $25.9 \%$ (37/143 patients). Lymph nodes $(n=5,079)$ were dissected, and 98 (1.9\%) were histologically confirmed metastatic lymph nodes.

The mean $\mathrm{SUV}_{\text {max }}, \mathrm{SUV}_{\text {mean }}, \mathrm{MTV}$, and TLG of the primary tumors of all patients were $6.6 \pm 6.7$ (range, 1.0-39.8), $2.7 \pm 1.9$ (range, 1.0-15.0), $12.0 \pm 21.3 \mathrm{~cm}^{3}$ (range, $0.0-120.0 \mathrm{~cm}^{3}$ ), and $994.7 \pm 2,350.7$ (range, 0.0-13,108.8), respectively. Differences
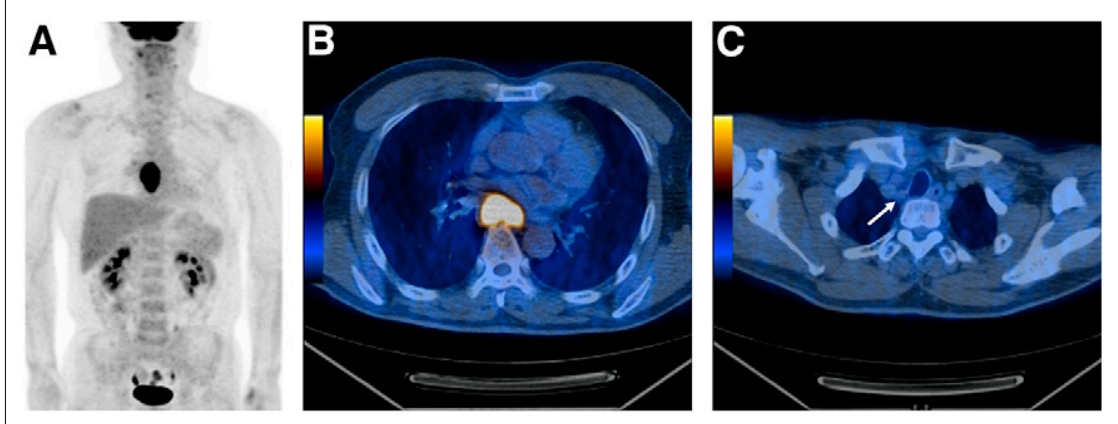

FIGURE 1. Images of a 60 -y-old male patient with newly diagnosed esophageal cancer. (A) Maximum-intensity-projection image shows hypermetabolic mass in mid to lower thoracic esophagus area. (B) Transverse image of PET/CT scan obtained at subcarinal level shows hypermetabolic mass with high SUV $V_{\max }$ of 14.7 in esophagus. No significant hypermetabolic or enlarged lymph nodes suggestive of metastasis were noted in staging work-up. (C) Small-sized right recurrent laryngeal lymph node without significant ${ }^{18} \mathrm{~F}$-FDG uptake is observed (arrow). After esophagectomy with node dissection, lymph node was found to be metastatic. in the metabolic parameters between the patients with and without occult lymph node metastasis are presented in Table 2 . The values for $\mathrm{SUV}_{\text {max }}, \mathrm{SUV}_{\text {mean }}$, and MTV of the primary tumor in patients with occult lymph node metastasis were significantly higher than those of the primary tumor in patients without occult lymph node metastasis (Fig. 1), whereas TLG of the primary tumor was not significantly different between the 2 groups.

\section{Risk Factors for Occult Lymph Node Metastasis}

Univariate analysis indicated that clinical $\mathrm{T}$ classification, $\mathrm{SUV}_{\max }$, $\mathrm{SUV}_{\text {mean }}, \mathrm{MTV}, \mathrm{TLG}$, and longitudinal diameter of tumors were significant risk factors associated with occult lymph node metastasis. Optimal thresholds were cT2-4, $\mathrm{SUV}_{\text {max }} \geq 4.8, \mathrm{SUV}_{\text {mean }} \geq 3.2$, MTV $\geq 5.5 \mathrm{~cm}^{3}$, TLG $\geq 220$, and diameter $\geq 3.8 \mathrm{~cm}$ (Table 3 ). In multivariate analysis without a combination of clinical $\mathrm{T}$ classification and $\mathrm{SUV}_{\max }$, clinical $\mathrm{T}$ classification and $\mathrm{SUV}_{\max }$ were independent risk factors (Table 4). With a combination of clinical $\mathrm{T}$ classification and $\mathrm{SUV}_{\max }, \mathrm{cT} 2-4$ and $\mathrm{SUV}_{\max } \geq 4.8$ was the only independent risk factor for occult lymph node metastasis.

In subgroup analysis, there was no significant risk factor in the cT1N0 group. However, in the cT2N0 group, $\operatorname{SUV}_{\max }(\geq 4.8)$ was the only risk factor (hazard ratio, 9.0; 95\% confidence interval, 1.7-48.4; $P=0.011)$.

Predicting performance of the selected risk factors for occult lymph node metastasis is summarized in Table 5. The combination of clinical T classification (cT2-4) and $\mathrm{SUV}_{\max }(\geq 4.8)$ was the most reliable parameter.

\section{Patterns of Occult Lymph Node Metastasis According to Primary Tumor Location}

The regions of occult lymph node metastasis according to the primary tumor location are summarized in Supplemental Table 1 (available at http://jnm.snmjournals.org).

Of 5 patients with upper thoracic esophageal cancer, 4 showed occult lymph node metastasis in the cervical area or upper mediastinum $(80.0 \%)$. Only 1 patient had skip metastasis in the upper abdomen (20.0\%). Recurrent laryngeal lymph nodes were most commonly involved (71.4\%, 5/7 lymph nodes).

Among 18 patients who had a primary tumor on the middle thoracic esophagus, 8 showed occult lymph node metastasis only in the mediastinum (44.4\%), whereas another 8 had occult lymph node metastasis in both the mediastinum and the upper abdomen (44.4\%) and 2 had metastasis only in the abdominal region (11.1\%). The tumors most commonly involved the left gastric lymph node $(37.8 \%, 17 / 45$ lymph nodes) in these patients.

Of 14 patients with a primary tumor on the lower thoracic esophagus or esophagogastric junction, 5 patients showed occult lymph node metastasis only in the upper abdomen $(35.7 \%)$. Another 4 patients had metastasis in both the mediastinum and the upper abdomen (28.6\%), and the others had metastasis only in the mediastinum $(35.7 \%)$. The left gastric lymph node was most commonly involved $(33.3 \%, 14 / 42$ lymph nodes). Importantly, almost half of the patients in whom the primary tumor was located in the lower thoracic esophagus or esophagogastric junction had occult lymph node metastasis involving the upper mediastinum $(42.8 \%, 6 / 14$ patients). 
TABLE 3

Univariate Analysis of Risk Factors Associated with Occult Lymph Node Metastasis

\begin{tabular}{|c|c|c|c|c|}
\hline Variable & Category & Hazard ratio & $95 \%$ confidence interval & $P$ \\
\hline cT classification & cT2-4 & 8.4 & $3.5-20.3$ & $<0.001$ \\
\hline $\mathrm{SUV}_{\max }$ & $\geq 4.8$ & 7.2 & $3.1-16.9$ & $<0.001$ \\
\hline $\operatorname{MTV}\left(\mathrm{cm}^{3}\right)$ & $\geq 5.5$ & 6.3 & $2.5-15.6$ & $<0.001$ \\
\hline TLG & $\geq 220$ & 5.8 & $2.3-14.4$ & $<0.001$ \\
\hline SUV $_{\text {mean }}$ & $\geq 3.2$ & 5.0 & $2.3-11.1$ & $<0.001$ \\
\hline cLongitudinal diameter of tumor $(\mathrm{cm})$ & $\geq 2.5$ & 2.2 & $1.0-4.9$ & 0.044 \\
\hline Age $(y)$ & $\geq 77$ & 3.2 & $0.9-11.6$ & 0.084 \\
\hline Sex & Male & 4.2 & $0.5-33.5$ & 0.179 \\
\hline \multirow[t]{4}{*}{ Tumor location } & Upper & & & 0.329 \\
\hline & Middle & 2.0 & $0.1-29.8$ & 0.615 \\
\hline & Lower & 0.6 & $0.1-6.7$ & 0.655 \\
\hline & Esophagogastric junction & 0.7 & $0.1-8.9$ & 0.815 \\
\hline \multirow[t]{3}{*}{ Histologic grade } & Well & 0 & & 0.962 \\
\hline & Moderate & 1.2 & $0.2-6.8$ & 0.827 \\
\hline & Poor & 1.2 & $0.3-4.7$ & 0.782 \\
\hline
\end{tabular}

$\mathrm{c}=$ clinical

\section{DISCUSSION}

The present study demonstrates that $\mathrm{SUV}_{\max }$ of the primary tumor and cT classification are independent risk factors associated with occult lymph node metastasis in patients with clinically NO esophageal squamous cell carcinoma. Our data further suggest that $\mathrm{SUV}_{\max }$ might be useful in identifying patients at risk of occult lymph node metastasis who should be managed more carefully.

Occult lymph node metastasis is important because it hinders exact assessment of the tumor status and selection of an appropriate treatment strategy. In addition, occult lymph node metastasis is itself a significant prognostic factor in patients with esophageal cancer (3). However, despite comprehensive assessment using multiimaging modalities, preoperative nodal staging work-up with conventional methods has a considerable rate of false-negative results. In this study, the rate of occult lymph node metastasis was $25.9 \%(37 / 143)$, which is consistent with previous studies (11\%-56\%) (3).

The diagnostic sensitivity of imaging modalities for detecting nodal involvement in clinically N0 patients is limited. PET/CT could not improve the detection rate of subclinical lymph node metastasis in the previous study (13). EUS with fine-needle aspiration has an excellent sensitivity in detecting the locoregional node metastasis of esophageal cancer (14); however, concerns exist regarding the interobserver variation and machine-dependent factors of EUS (15). In addition, patient cooperation is essential, therefore safe and successful EUS is not practical in all patients. In these circumstances, knowledge of risk factors related with occult lymph node metastasis could improve our perception of node metastasis.

Various characteristics of esophageal cancer have been reported as important factors for lymph node metastasis $(11,13-15)$. Immunohistochemical findings, such as depth of tumor infiltration and grade of lymphocytic infiltration, are important factors in early esophageal cancer $(11,14,15)$. However, before endoscopic resection or esophagectomy, accurate immunohistochemical factors related to lymph node metastasis could not be obtained. In practice, histology obtained from endoscopic biopsies, tumor size or depth determined by preoperative evaluation, and other clinical characteristics of patients constitute available information for the prediction of occult lymph node metastasis. To date, however, risk factors for the prediction of occult lymph node metastasis in patients with esophageal cancer have not been fully investigated.

The presence of muscularis propria invasion determined by EUS (cT2) is an independent risk factor associated with occult lymph node metastasis in this study, corresponding with the earlier studies that reported that endoscopically determined clinical tumor depth was a significant independent factor for predicting lymph node metastasis in esophageal cancer $(13,16)$. In addition, cT classification with $\mathrm{SUV}_{\max }(\geq 4.8)$ is a powerful parameter predicting occult lymph node metastasis (Tables 4 and 5). In patients undergoing esophagectomy, the primary tumor with muscularis propria invasion with high $\mathrm{SUV}_{\max }$ may justify extensive and careful regional lymph node dissection, especially in the upper mediastinum

TABLE 4

Multivariate Analysis of Risk Factors Associated with Occult Lymph Node Metastasis

\begin{tabular}{|c|c|c|c|c|}
\hline Variable & Category & Hazard ratio & 95\% Confidence interval & $P^{\star}$ \\
\hline cT classificationt & cT2-4 & 4.6 & $1.7-12.4$ & 0.003 \\
\hline $\mathrm{SUV}_{\max }^{\dagger}$ & $\geq 4.8$ & 3.5 & $1.3-09.2$ & 0.012 \\
\hline cT classification + SUV $_{\max }$ & cT2-4 and $\geq 4.8$ & 13.2 & $5.4-31.9$ & $<0.001$ \\
\hline $\begin{array}{l}{ }^{\star} \text { Adjusted for age and sex. } \\
{ }^{\dagger} \text { Obtained from multivariate } \\
\mathrm{C}=\text { clinical. }\end{array}$ & kcluding cT class & $-\mathrm{SUV}_{\max }$ & & \\
\hline
\end{tabular}


TABLE 5

Predicting Performance of Selected Parameters for Occult Lymph Node Metastasis

\begin{tabular}{|c|c|c|c|c|c|}
\hline Variable & Sensitivity & Specificity & Positive predictive value & Negative predictive value & Accuracy \\
\hline cT classification & $78.4 \%$ & $69.8 \%$ & $47.5 \%$ & $90.2 \%$ & $72.0 \%$ \\
\hline $\mathrm{SUV}_{\max }$ & $75.7 \%$ & $69.8 \%$ & $46.7 \%$ & $89.2 \%$ & $71.3 \%$ \\
\hline cT classification $+S_{\text {max }}$ & $73.0 \%$ & $81.5 \% *$ & $60.0 \%$ * & $89.7 \%$ & $80.4 \%{ }^{*}$ \\
\hline $\begin{array}{l}{ }^{*} P<0.001, \text { compared to } \mathrm{cT} \\
\mathrm{C}=\text { clinical. }\end{array}$ & ssification & SUV $_{\text {max }}$ & & & \\
\hline
\end{tabular}

and abdomen, due to the probability of occult metastasis (Supplemental Table 1).

To the best of our knowledge, the predictive value of PET parameters for postoperative nodal status in clinically N0 squamous cell carcinoma of the esophagus has not been established. However, we could reasonably infer that PET parameters may be related to postoperative nodal involvement in clinically N0 patients because the previous studies have shown that PET parameters are positively correlated with $\mathrm{N}$ classification in various cancers $(8$, 17-19). $\mathrm{SUV}_{\max }$ of the primary tumor was significantly associated with postoperative nodal status in patients with clinically NO nonsmall cell lung cancer (17). $\mathrm{SUV}_{\max }>12.7$ and average $\mathrm{SUV}>$ 5.9 yielded the best result to predict nodal metastases in a study population consisting of patients with mainly adenocarcinoma of the esophagus (19). Results of our study are in close agreement with those of previous studies. Our findings and those of others suggest that physicians should be careful to establish node status when they deal with an esophageal cancer patient who has a high $\mathrm{SUV}_{\max }$ of the primary tumor even when the size and ${ }^{18} \mathrm{~F}$-FDG uptake of lymph nodes may not be significant enough to suggest metastasis in preoperative staging.

In our institution, cTis-T1N0 esophageal cancer patients underwent esophagectomy or endoscopic mucosal resection without preoperative chemoradiotherapy, and cT1N-positive esophageal cancer patients mainly underwent preoperative chemoradiotherapy according to the recommendations of the guideline (20). In the cT1N0 group, there were no significant risk factors related with occult lymph node metastasis, which may be partly explained by a low event rate of occult lymph node metastasis in the cT1N0 group $(12.3 \% ; 8 / 65)$. This finding suggests that PET parameters do not affect the decision-making process in cT1N0 disease. On the other hand, in other groups including cT2N0, SUV $\max$ (cutoff, 4.8) was the only significant risk factor associated with occult lymph node metastasis. In cT2-4aN0 esophageal cancer, there are various primary treatment options including preoperative chemoradiotherapy, definitive chemoradiation, and esophagectomy (20). Our results suggest that $\mathrm{SUV}_{\max }$ may be useful for choosing appropriate primary treatment in those groups. For example, in the case of low-risk cT2-4a disease, esophagectomy is the preferred option. If the primary tumor has an $\mathrm{SUV}_{\max } \geq 4.8$, preoperative chemoradiotherapy might be the more appropriate primary treatment because of a high risk of occult lymph node metastasis.

Theoretically, volume-based parameters are more suitable indicators of tumor burden than $\mathrm{SUV}_{\text {max }}$ or $\mathrm{SUV}_{\text {mean }}$, because volumebased PET parameters have shown a better predictive performance for prognosis than $\mathrm{SUV}_{\max }$ in previous studies (21-23). In addition, MTV demonstrated a significant correlation with occult lymph node metastasis in patients with clinically N0 tongue cancer (8). However, the present study revealed that $\mathrm{SUV}_{\text {max }}$, but not MTV or
TLG, is associated with occult lymph node metastasis. This finding suggests that the presence of malignant cells with high glucose metabolism is more important than total tumor burden in the process of lymph node metastasis in esophageal cancer.

The number of dissected lymph nodes is an important factor that might affect the results of the present study. If the number of dissected lymph nodes is insufficient, occult lymph node metastasis might be missed, resulting in an inaccurate result (24). The optimal number of nodes that should be dissected was reported in previous studies as at least $6-18$ lymph nodes in patients with early esophageal cancer (24-26). In the present study, an average of 36 lymph nodes (range, 6-108) was dissected, which was a sufficient number to conduct an accurate $\mathrm{N}$ classification.

The pattern of occult lymph node metastasis was also investigated in this study. Patients with tumors located on the upper thoracic esophagus commonly had recurrent laryngeal lymph node metastasis. Patients with tumors located on the mid to lower thoracic esophagus or esophagogastric junction typically had left gastric lymph node metastasis. Most importantly, occult lymph node metastasis was not limited to the vicinity of the primary tumor: in $45.9 \%$ of patients (17/37), node metastasis skipped the adjacent area of primary tumor. The findings of our study suggest that lymph node dissection should be performed carefully and with sufficient range in patients at high risk of occult lymph node metastasis.

This study has several limitations. The retrospective nature of the study limits external validity of study findings. Generalization and clinical application to other populations and settings may be difficult. Another limitation is that possible risk factors, such as endoscopic findings, were not included in the analysis. The other limitation is that cT3-4 esophageal cancer patients were enrolled in this study. Because of current management guidelines, it is difficult to include these patients as preoperative chemoradiotherapy before esophagectomy is the preferred treatment for cT3-4 esophageal cancer. However, this therapeutic strategy has been established over the years. Until 2008, induction therapy was not routinely recommended in our institution. Consequently, $30(21 \%$; 30/143) patients who had cT3-4 esophageal cancer were included because we enrolled patients between 2003 and 2010. Further well-designed prospective studies are needed to confirm the clinical value of metabolic PET parameters in predicting occult lymph node metastasis.

\section{CONCLUSION}

The present study demonstrated that $\mathrm{SUV}_{\max }$, combined with clinical $\mathrm{T}$ classification, can be useful for predicting occult lymph node metastasis in patients with clinically NO squamous cell carcinoma of esophagus. These parameters may allow the identification of patients at high risk of occult lymph node metastasis who might be candidates for more careful diagnostic work-up and more intensive treatment. 


\section{DISCLOSURE}

The costs of publication of this article were defrayed in part by the payment of page charges. Therefore, and solely to indicate this fact, this article is hereby marked "advertisement" in accordance with 18 USC section 1734 . This study was supported by a grant from the National R\&D Program for Cancer Control, Ministry of Health and Welfare, Republic of Korea (1120150). No other potential conflict of interest relevant to this article was reported.

\section{REFERENCES}

1. Stahl M, Budach W, Meyer HJ, Cervantes A. Esophageal cancer: clinical practice guidelines for diagnosis, treatment and follow-up. Ann Oncol. 2010;21(suppl 5): v46-v49.

2. Sgourakis G, Gockel I, Lyros O, Hansen T, Mildenberger P, Lang H. Detection of lymph node metastases in esophageal cancer. Expert Rev Anticancer Ther. 2011; 11:601-612.

3. McGuill MJ, Byrne P, Ravi N, Reynolds J. The prognostic impact of occult lymph node metastasis in cancer of the esophagus or esophago-gastric junction: systematic review and meta-analysis. Dis Esophagus. 2008;21:236-240.

4. Fletcher JW, Djulbegovic B, Soares HP, et al. Recommendations on the use of ${ }^{18}$ F-FDG PET in oncology. J Nucl Med. 2008;49:480-508.

5. Liu FY, Chao A, Lai CH, Chou HH, Yen TC. Metabolic tumor volume by ${ }^{18} \mathrm{~F}-$ FDG PET/CT is prognostic for stage IVB endometrial carcinoma. Gynecol Oncol. 2012;125:566-571.

6. Hatt M, Visvikis D, Albarghach NM, Tixier F, Pradier O, Cheze-le Rest C. Prognostic value of ${ }^{18} \mathrm{~F}$-FDG PET image-based parameters in oesophageal cancer and impact of tumour delineation methodology. Eur J Nucl Med Mol Imaging. 2011;38:1191-1202.

7. Wahl RL, Jacene H, Kasamon Y, Lodge MA. From RECIST to PERCIST: evolving considerations for PET response criteria in solid tumors. J Nucl Med. 2009;50(suppl 1):122S-150S.

8. Chung MK, Jeong HS, Son YI, et al. Metabolic tumor volumes by $\left[{ }^{18} \mathrm{~F}\right]-$ fluorodeoxyglucose PET/CT correlate with occult metastasis in oral squamous cell carcinoma of the tongue. Ann Surg Oncol. 2009;16:3111-3117.

9. Kanzaki R, Higashiyama M, Fujiwara A, et al. Occult mediastinal lymph node metastasis in NSCLC patients diagnosed as clinical N0-1 by preoperative integrated FDG-PET/CT and CT: risk factors, pattern, and histopathological study. Lung Cancer. 2011;71:333-337.

10. Moon SH, Choi JY, Lee HJ, et al. Prognostic value of ${ }^{18} \mathrm{~F}-\mathrm{FDG}$ PET/CT in patients with squamous cell carcinoma of the tonsil: comparisons of volumebased metabolic parameters. Head Neck. 2013;35:15-22.

11. Kim DU, Lee JH, Min BH, et al. Risk factors of lymph node metastasis in T1 esophageal squamous cell carcinoma. J Gastroenterol Hepatol. 2008;23:619-625.
12. Huang W, Li B, Gong H, et al. Pattern of lymph node metastases and its implication in radiotherapeutic clinical target volume in patients with thoracic esophageal squamous cell carcinoma: a report of 1077 cases. Radiother Oncol. 2010;95:229-233.

13. Gaur P, Sepesi B, Hofstetter WL, et al. A clinical nomogram predicting pathologic lymph node involvement in esophageal cancer patients. Ann Surg. 2010;252:611-617.

14. Ancona E, Rampado S, Cassaro M, et al. Prediction of lymph node status in superficial esophageal carcinoma. Ann Surg Oncol. 2008;15:3278-3288.

15. Xue L, Ren L, Zou S, et al. Parameters predicting lymph node metastasis in patients with superficial esophageal squamous cell carcinoma. Mod Pathol. 2012;25: 1364-1377.

16. Bollschweiler E, Baldus SE, Schroder W, et al. High rate of lymph-node metastasis in submucosal esophageal squamous-cell carcinomas and adenocarcinomas. Endoscopy. 2006;38:149-156.

17. Zhu WQ, Sun X, Xing L, et al. Oesophageal squamous cell carcinoma: relationship between fluorine-18 fludeoxyglucose positron emission tomography CT maximum standardised uptake value, metabolic tumour volume, and tumour, node and metastasis classification. Br J Radiol. 2012;85:e383-e387.

18. Miyasaka Y, Suzuki K, Takamochi K, Matsunaga T, Oh S. The maximum standardized uptake value of fluorodeoxyglucose positron emission tomography of the primary tumour is a good predictor of pathological nodal involvement in clinical N0 non-small-cell lung cancer. Eur J Cardiothorac Surg. 2013;44:83-87.

19. Mamede M, Abreu ELP, Oliva MR, Nose V, Mamon H, Gerbaudo VH. FDG$\mathrm{PET} / \mathrm{CT}$ tumor segmentation-derived indices of metabolic activity to assess response to neoadjuvant therapy and progression-free survival in esophageal cancer: correlation with histopathology results. Am J Clin Oncol. 2007;30:377-388.

20. National Comprehensive Cancer Network. Ajani JA, Barthel JS, Bentrem DJ, et al. Esophageal and esophagogastric junction cancers: NCCN Clinical Practice Guidelines in Oncology—v.2.2013. May 6, 2013. http://www.nccn.org/professionals/ physician_gls/f_guidelines.asp\#esophageal. Accessed March 6, 2014.

21. Hyun SH, Choi JY, Kim K, et al. Volume-based parameters of ${ }^{18} \mathrm{~F}$-fluorodeoxyglucose positron emission tomography/computed tomography improve outcome prediction in early-stage non-small cell lung cancer after surgical resection. Ann Surg. 2013;257:364-370.

22. Hyun SH, Choi JY, Shim YM, et al. Prognostic value of metabolic tumor volume measured by ${ }^{18} \mathrm{~F}$-fluorodeoxyglucose positron emission tomography in patients with esophageal carcinoma. Ann Surg Oncol. 2010;17:115-122.

23. Yoo J, Choi JY, Lee KT, et al. Prognostic significance of volume-based metabolic parameters by ${ }^{18} \mathrm{~F}-\mathrm{FDG}$ PET/CT in gallbladder carcinoma. Nucl Med Mol Imaging. 2012;46:201-206.

24. Hu Y, Hu C, Zhang H, Ping Y, Chen LQ. How does the number of resected lymph nodes influence TNM staging and prognosis for esophageal carcinoma? Ann Surg Oncol. 2010;17:784-790.

25. Greenstein AJ, Litle VR, Swanson SJ, Divino CM, Packer S, Wisnivesky JP. Effect of the number of lymph nodes sampled on postoperative survival of lymph node-negative esophageal cancer. Cancer. 2008;112:1239-1246.

26. Rizk NP, Ishwaran H, Rice TW, et al. Optimum lymphadenectomy for esophageal cancer. Ann Surg. 2010;251:46-50. 